presented as an academic history then the lack of exploration of more sources including archive material from the meetings and correspondence from Harvard's biology department and Cold Spring Harbor's board of trustees - would be a concern. But as a kind of Festschrift, this volume should encourage as well as inform such a work. It will also entertain.

Inspiring Science could be read alongside Victor McElheny's biography of our subject, Watson and DNA (for a review see Nature 421, 315-316; 2003). The editors are to be congratulated on compiling and organizing such a readable and informative set of recollections that will serve very effectively the role they intended.

Robert Olby is in the Department of the History and Philosophy of Science, University of Pittsburgh, Pittsburgh, Pennsylvania 15260, USA.

\section{An inside view of mental health}

\section{The Confinement of the Insane: International Perspectives, 1800-1965 \\ edited by Roy Porter \& David Wright Cambridge University Press: 2003. 371 pp.

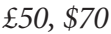

\section{Gerald N. Grob}

The history of mental illness and psychiatry before 1960 was written within a Whiggish tradition of inevitable progress, with new psychiatric knowledge and therapies superseding ignorance and barbarism. The subsequent failure of mental hospitals to live up to this promise was attributed to shortsighted and parsimonious public policies that had led to overcrowding and neglect of therapeutic functions.

After 1960, by contrast, the history of psychiatry and mental hospitals underwent fundamental changes. A group of social and psychiatric critics launched an attack on the legitimacy of both psychiatry and mental hospitals and led the development of a quite different synthesis. In so doing they used what purported to be empirical data to support their interpretations. In Michel Foucault's eyes, for example, mental hospitals were institutions of social control that served the interests of a capitalist society that prized productivity above all else. Others, including Thomas Szasz, Erving Goffman and Thomas Scheff, emphasized the repressive nature of such institutions, and some critics even challenged the very existence of mental disorders.

The work of these critics influenced a group of historians who then developed a synthesis that was equally critical of psychiatry and mental hospitals. They emphasized élite hegemony: people with mental illnesses were simply objects without agency. The confinement of the mentally ill in institutions reflected fears of social disorder and a desire to penalize those who did not contribute to productivity. This scholarship in some ways symbolized the triumph of ideology over reality, as their authors ignored a large body of contradictory data.

In recent years a new and different synthesis has begun to emerge. The Confinement of the Insane, a collection of essays by younger scholars that cover developments in no less than fourteen countries on five continents, is but one indication of new trends. Dealing with the institutions and policies of countries with differing populations, traditions and cultures, these scholars largely eschew the angry and polemical writings of the 1960s and 1970s. Basing their analyses on archival data, they present nuanced and subtle interpretations that offer fresh insight into the mental-health policies of different nations.

The authors of these essays avoid a historical analysis that assumes inevitable progress. Yet they find that many of the allegations made by the psychiatric critics lack substance. In their eyes, psychiatry was not a profession characterized solely by selfinterest, nor were mental hospitals merely places of confinement for inconvenient people. In place of simplicity, these younger historians offer a complex model that grows out of deep research. They reject the claim that mental hospitals were created by hegemonyseeking élites, and insist that they were multifaceted institutions that simultaneously served caring, therapeutic and custodial functions. Indeed, these institutions were contested arenas where families, patients, public officials and psychiatrists negotiated among themselves. Admission to the mental hospital, for example, was rarely controlled by police or officials concerned with the maintenance of public order; in most countries, families played key roles in institutionalizing their members.

Moreover, the authors' research undermines older generalizations about the composition of hospital populations. Hospital stays in nineteenth-century England, for example, were relatively brief. In most places, unmarried individuals and males had the highest rates of being institutionalized; females had lower rates, but once inside they tended to remain there longer. The oft-repeated claim that women had disproportionately high rates of incarceration is not supported by empirical data.

Nor were hospitals populated by the fringe elements of industrial society. The analysis of Canadian hospitals, for example, found that most patients were productively employed. Finally, the comparative dimensions of these essays suggests that indigenous cultures and traditions played significant roles in shaping different mental-health policies. These and other data do little to support the claims of ideologically driven scholars that mental hospitals were places where inconvenient and marginal people were incarcerated.

The essays in this volume do not lead to overarching interpretations, but nevertheless they highlight the importance of using patient records and avoiding ideological clichés. Such research can also have contemporary policy implications. Knowledge

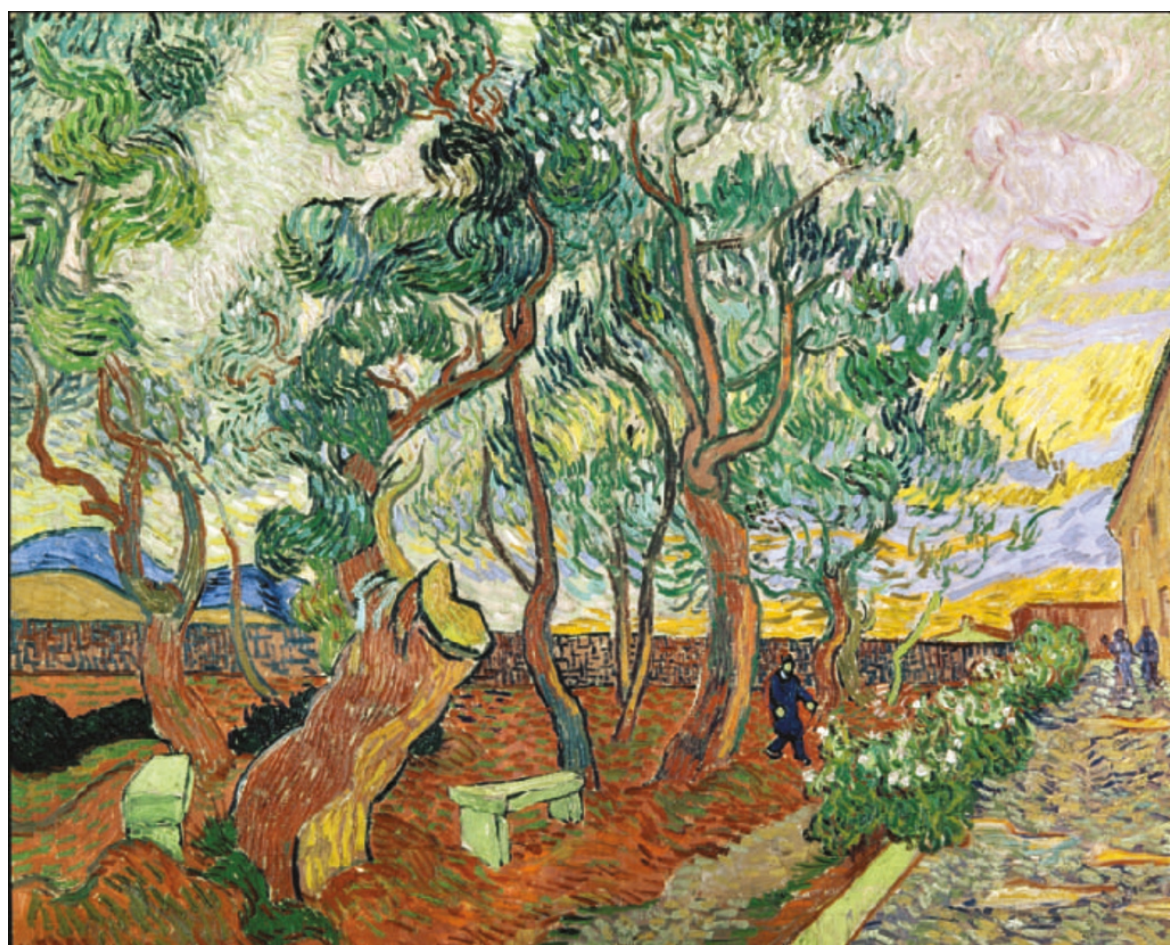

Vincent van Gogh painted The Garden of St Paul's Hospital at St Remy during a spell in the asylum. 


\section{Making a case for life}

Exhibits at the Natural History Museum in London, such as these models of rat fleas on show in 1927, have been drawing the crowds since the museum opened its doors in 1881. This exhibit was part of a campaign to inform the public of the role of insects in spreading disease.

Photographers, both professional and amateur, have recorded events in the life of 'the world's greatest cathedral to natural history', which has amassed a collection of more than 6,000 images. As well as showing the public face of the museum, the collection contains many pictures showing the minutiae of daily life behind the scenes, as the staff worked to conserve and prepare the exhibits. Arrivals and departures of famous specimens such as the blue whale are recorded, with the style of exhibits changing along with the hairstyles and dress of visitors and staff.

About 100 of these images are themselves now on show, in an exhibition that runs until 20 February 2004 and in a book, Life Through a Lens by Susan Snell and Polly Tucker (Natural History Museum Publishing, £15).

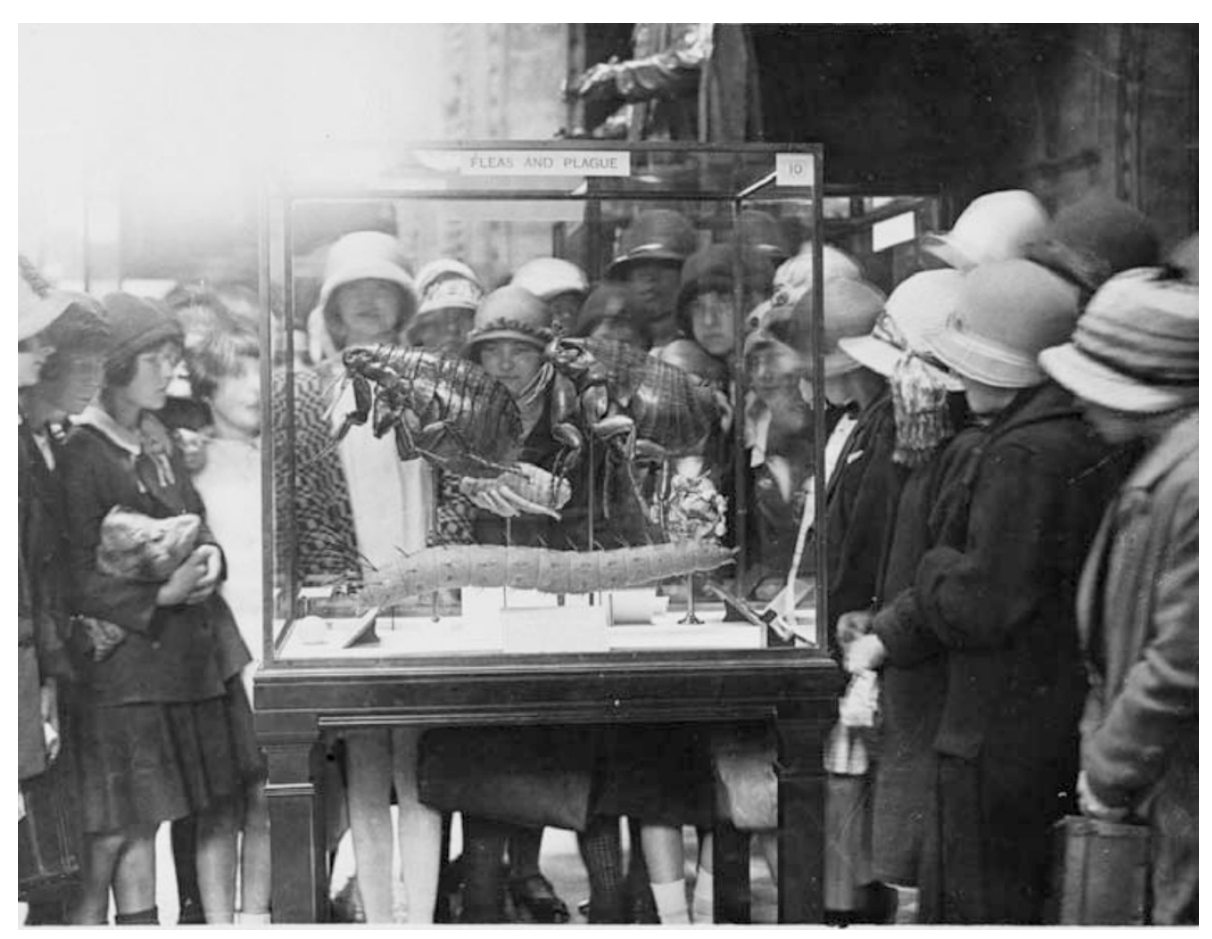

about the past does not necessarily point the way to future action, but it can serve as an important corrective to misperceptions about the past, which have frequently led to policy-making based on ideology rather than reality.

Gerald N. Grob is emeritus professor at the Institute for Health, Health Care Policy, and Aging Research, Rutgers University, 30 College Avenue, New Brunswick, New Jersey 08901, USA.

\section{Core research}

\section{The Dynamic Structure of the Deep Earth: An Interdisciplinary Approach}

by Shun-ichiro Karato

Princeton University Press: 2003. 264 pp.

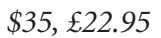

\section{Quentin Williams}

The deep interior of the Earth is, like space, a seemingly endless source of inspiration for popular fiction. A recent movie, The Core, followed this tried-and-tested theme with a tale of travel to the centre of the planet. Along the way, a predictable sequence of characters must die (with their order of demise apparently determined by development, ethnicity, nationality and personality) to ensure that the outrageously attractive protagonists survive to return to the surface.

The realities associated with the deep Earth are actually far more interesting than one might guess from Hollywood's formulaic prosaism. The deep Earth manufactures diamonds and delivers them, uncombusted, to Earth's surface. Its degassing probably furnished us with Earth's surface water and the starting materials for our atmosphere. And it provides the engine for plate tectonics - the source of mountains, ocean basins and almost everything in between.

Our understanding of Earth's interior has mushroomed over the past two decades, driven by a synergy between improved seismic imaging, better understanding of the properties of Earth's materials at extreme pressures and temperatures, and computationally intensive simulations of the planet's interior.

The author of The Dynamic Structure of the Deep Earth, Shun-ichiro Karato, was trained in rock mechanics and has worked on a broad range of deep-Earth related topics, so he is well equipped to write such a book. Its intent, as outlined in the preface, is not to provide a synopsis, but rather to introduce the interdisciplinary approach of the subtitle through specific problems on which the author has worked. The book seems to belong to a fine tradition of volumes about the deep Earth that incorporate general introductions to research areas but also serve to revisit and remind people of the (mostly already published) viewpoints of the author.

This style perhaps reached its apex with A. E. Ringwood's Origin of the Earth and Moon (Springer, 1979), which both placed Ringwood's views into a tutorial framework and provided a focal point for scientific disagreements. This is a lofty benchmark, and the ultimate success of Karato's book will be determined by the long-term importance and longevity of the views described.

The topics of the book span the depth range of the planet, from the boundary between the rigid outer lithosphere and the underlying fluid asthenosphere to the inner core. The introductory chapter is one of the highlights of the book - a nice pedagogic overview of Earth's structure, complete with boxes containing derivations of the relevant parameters. The author then provides reviews and gives his own take on a selection of current deep-Earth research problems. These include discussions of the lithosphereasthenosphere boundary and the origin of the 'low-velocity zone' within the shallow upper mantle; the meaning of seismically fast and slow zones in tomographic models of Earth's interior; the relationship between

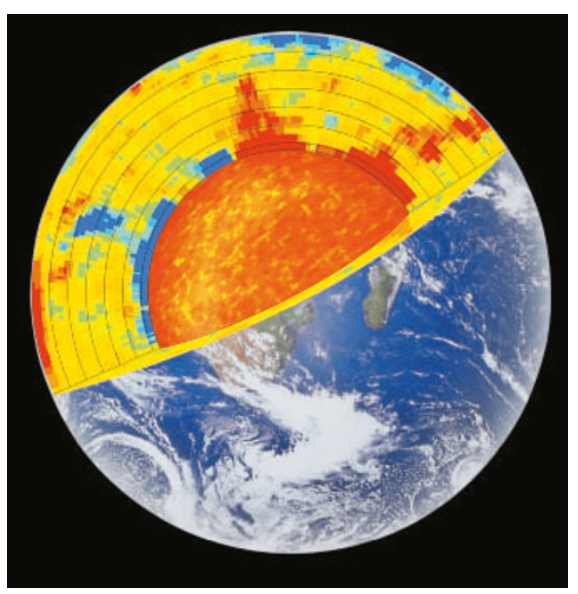

The rise and fall of slabs of hot (red) and cold (blue) rock in the mantle drives plate tectonics. 